\title{
Medizinstudium und Arztberuf
}

\author{
Marco Mumenthaler gehört zweifellos zu den herausragenden Arzt- und Me- \\ dizinerpersönlichkeiten in unserem Land. Wir freuen uns deshalb darüber, für \\ unsere Leserschaft zum Auftakt des neuen Jahres die bemerkenswerte Rede \\ zu Medizinstudium und Arztberuf wiedergeben zu können, die er anlässlich \\ der Staatsexamensfeier 2006 im Zürcher Grossmünster gehalten hat.
}

Die Redaktion

Marco Mumenthaler

Korrespondenz:

Prof. Dr. med. Marco Mumenthaler Facharzt für Neurologie FMH

Witikonerstrasse 326

CH-8053 Zürich

Tel./Fax 0443817685

mumenthaler33@bluewin.ch
Liebe Absolventinnen und Absolventen des diesjährigen Staatsexamens,

liebe junge Kolleginnen und Kollegen, vir spectabilis, meine Damen und Herren

Wenn ich zu Ihnen, liebe Staatsexamensabsolventinnen und -absolventen sprechen darf, dann als jemand, der Ihren bisherigen Weg und auch den zukünftigen Weg der meisten unter Ihnen ebenfalls schon gegangen ist: ein Studium der Medizin, eine Weiterbildung zum Spezialarzt, eine Dozenten- und Chefarzt-Karriere sowie eine praktische ärztliche Tätigkeit in verschiedenen Kontinenten und Ländern bis hin zum Irak.

Aber nun zum eigentlichen Thema: Studium und Beruf. Das Studium, diese erste Phase im Rahmen der Ausbildung, haben Sie hinter sich. Einen langen Lebensweg haben Sie noch vor sich. Ich habe die Zeit meines eigenen Medizinstudiums in drei verschiedenen europäischen Ländern und an vier Universitäten in sehr lebendiger Erinnerung.

- Haben auch Sie die Diskrepanz zwischen der Verpflichtung, Wissen zu speichern, und der mangelnden Einsicht in die Nützlichkeit dieses Wissens schmerzlich erlebt?

- Waren auch Sie oft ernüchtert von der gelangweilten Schnoddrigkeit, mit welcher manche Ihrer Lehrer den Stoff vorgetragen haben zum Glück nicht alle.

- Haben auch Sie oft resigniert ob der fast unvermeidlichen Anonymität, welche in den überfüllten Hörsälen herrscht?

- Waren Sie nicht auch befremdet, wenn ein Dozent kühl bis farblos seinen Stoff vortrug und Sie in ihm keine Begeisterung spürten?

- Waren Sie nicht in Ihrem tiefsten Inneren enttäuscht, wenn Ihnen zwar Wissensvermittler entgegentraten, selten aber Persönlichkeiten und kaum je Vorbilder?
Im Laufe der Jahre wurde es für mich immer klarer, dass im Studium bis zum Schlussexamen der junge Mediziner folgendes wünscht und braucht:

- Ein solides Grundwissen, ohne Verirrungen in allzu spezialistische Details.

- Ein Grundwissen, auf welchem er in den folgenden Weiterbildungsjahren und in den Jahren der Berufsausübung aufbauen kann.

- Dann aber auch und vor allem braucht er Hinweise, um mit diesem Wissen am Krankenbett angemessen und sinnvoll umgehen zu können.

- Er braucht Vorbilder, die ihn beispielhaft miterleben lassen, wie er sein Wissen im einzelnen Fall differenziert anwenden kann;

- Vorbilder, die ihm die menschlichen Aspekte und die sozialen Dimensionen des Arztseins erleben lassen.

Ja, auch wenn vielleicht nicht alle diese Forderungen in Ihrem bisherigen Studium verwirklicht waren, so haben Sie alle das Medizinstudium, die Phase der Ausbildung erfolgreich nunmehr abgeschlossen. Dazu gratuliere ich Ihnen im Namen all Ihrer Lehrer von Herzen. Freuen Sie sich, und seien Sie stolz auf diesen Erfolg.

Apropos Lehrer: Ich möchte Ihnen verraten, zu welcher Überzeugung ich in bezug auf die Definition eines guten Lehrers im Laufe der Jahre gekommen bin: Ein guter Lehrer kann nur der sein, der

1. die Begeisterung für das, was er lehrt, in all seinen Lehrveranstaltungen spüren lässt, und

2. vor allem muss er jene, denen er etwas vermitteln will, von Herzen gern haben.

Und damit möchte ich nun zum zweiten Teil meiner Ansprache übergehen: zum Beruf. Sie 
werden jetzt, sofern Sie den Arztberuf im klassischen Sinne des Wortes ausüben wollen, eine Weiterbildung an Spitälern antreten. Sie lernen zwar weiterhin dazu, aber Sie üben als Assistenzärztin oder Assistenzarzt schon den Beruf recht eigentlich aus. Sie übernehmen also Verantwortung. Auf den Begriff der Verantwortung werden wir übrigens noch einmal zurückkommen.

Sie werden sich also für eine Fachrichtung innerhalb der Medizin entscheiden. Sie werden sich dabei bewusst werden, dass es fast unbegrenzte Möglichkeiten gibt, als diplomierter Arzt zu wirken. Dies geht vom Medizininformatiker über die Mitarbeit in einer Pharmafirma, der wissenschaftlichen Tätigkeit in einer chemischen Industrie, das Forschen an einem Universitätsinstitut, den Beruf eines Medizinjournalisten, eines Moderators eines Gesundheitsmagazins am Fernsehen, eines Mitarbeiters im Gesundheitssektor einer Behörde bis hin zum Regierungsrat eines schweizerischen Kantons, zu einem Dichter wie Friedrich Schiller, zu einem Revolutionär wie Che Guevara oder, für die Frauen unter Ihnen, zur Präsidentin eines südamerikanischen Staates. Aber die allermeisten unter Ihnen werden dann doch Doktor werden, den Beruf also ausüben, für den Sie eigentlich im Studium ausgebildet wurden. Sie werden also gewissermassen die praktisch angewandte Medizin ausüben.

Innerhalb der Fachgebiete werden Sie eines ergreifen, von der allgemeinen Medizin bis zu einer der mehr als 30 Spezialgebiete und Unterspezialitäten der Medizin in unserem Lande. Welches dieses Fachgebiet sein wird, hängt glauben Sie mir - in erster Linie vom Zufall ab, zum Beispiel von einer freien Stelle. Dann hängt es aber auch von Menschen ab, von Vorbildern. Wenn Sie ein Dozent oder eine Dozentin im Unterricht positiv beeindruckt hat, wenn Ihnen ein Oberarzt im Praktikum imponierte, werden Sie für das betreffende Fach vermehrt motiviert sein. Verzichten Sie darauf, hartnäckig eine bestimmte Spezialisierung à tout prix anzustreben, eine Spezialisierung, von der Sie meinen, dass es für Sie die einzig richtige sei. Sie werden mit Staunen feststellen, dass so gut wie jedes Spezialgebiet Ihnen mit der Zeit lieb werden wird. Es ist so, wie Leonardo da Vinci es ausgedrückt hat: «Il grande amore nasce dalla profonda conoscenza della cosa che si ama.» Und für jene unter Ihnen, welchen die italienische Landessprache nicht vertraut ist oder neben dem nicht gerade ein Tessiner sitzt, noch auf Deutsch: Die grosse Liebe erwächst erst aus der vertieften Kenntnis dessen, das man gerne hat. Dies gilt übrigens sowohl für ein Sachgebiet wie auch für einen Menschen.
Aber lassen wir das, wenden wir uns der grossen Mehrheit zu, die in die Praxis gehen wird. Dort werden Sie sich mit einer Reihe von Aspekten auseinandersetzen müssen. Ich will nur einige davon andeuten und Ihnen ein paar Ratschläge mitgeben: Es werden Patienten zu Ihnen kommen. Sie suchen sie sich nicht aus. Nicht alle sind Ihnen sympathisch. Und doch ist es Ihre Pflicht, jedem mit der gleichen Bereitschaft zu begegnen, ihm optimal beizustehen. Das bedeutet, dass Sie bereit sein müssen, jedem Patienten auf die für ihn geeignete Weise zu begegnen. Sie müssen angemessen mit dem türkischen Bauarbeiter umgehen, ebenso wie mit der Trottoiramsel von der Langstrasse, dem süffisanten Gymnasiasten vom Zürichberg oder mit dem sehr selbstbewussten Regierungsrat aus dem Kanton Aargau. Angemessen heisst aber, sich ihm in einer für ihn spezifisch spürbaren Weise zu öffnen, in einer für ihn spezifisch verständlichen Sprache mit ihm zu reden, sich also in sehr unterschiedliche Menschen einfühlen zu können und sie in sehr unterschiedlicher Weise Ihre Zuwendung spüren zu lassen. Der jüdische Arzt und Philosoph Mosche Ben Maimon hat im 12. Jahrhundert dies so formuliert: «Lass mich im Kranken stets nur den Menschen sehen, sei er mir nun lieb oder lästig, sei er gut oder schlecht, arm oder reich, mächtig oder unbedeutend.»

Ich versprach, noch etwas zur Verantwortung zu sagen. Ich hatte vorhin den Ausdruck Patienten gebraucht und nicht Klienten. Gewiss, der Patient wurde zumindest formal mündig und damit zum Klienten. Aber seien wir doch ehrlich: Den Vorsprung, den Sie als Ärztin oder als Arzt an Wissen und Überblick haben, ist trotz höherem Bildungsniveau unserer Bevölkerung und trotz Internet doch sehr beachtlich. Lassen Sie sich nicht durch falsch verstandene Partnerschaft mit Ihrem mündigen Patienten dazu verleiten, ihm die Verantwortung für das weitere Vorgehen alleine zu übertragen. Glauben Sie mir, die meisten Patienten sind Ihnen dankbar, wenn Sie ihm zumindest noch sagen würden, was Sie persönlich an seiner Stelle tun würden.

Mit diesen Sätzen haben wir indirekt auch die Macht des Arztes angesprochen. Mögen wir noch so bescheiden und einfühlsam mit dem uns vertrauenden Menschen umgehen, wir bleiben immer für den Kranken ein Mächtiger. Missbrauchen Sie diese Macht nicht. Lassen Sie den Patienten ruhig unseren Wissensvorteil spüren. Missbrauchen sie denselben jedoch nicht, sondern nutzen Sie den Vorteil dieser Stellung als ein Element Ihres therapeutischen Wirkens aus. 
Nochmals zu den Patienten, diesmal den schwierigen Patienten. Die gibt es, etwa den Patienten, der Ihnen mit offensichtlichem Misstrauen begegnet, der einen Ausdruck aus dem Internet vorlegt und wissen möchte, ob Ihnen die Therapie des Dr. Pesoa aus Saõ Paulo in Brasilien bekannt ist und was Sie davon halten. Der Patient also, der Sie masslos nervt. Sie dürfen genervt sein, Sie müssen es nur merken. Sie werden dann meist auf sachliche oder korrekte Distanz gehen und die Nervensäge sucht sich bald ein anderes Opfer. Oder aber, Sie überwinden aktiv Ihre berechtigte Abneigung, greifen auf Ihr besseres Ich zurück, sind betont freundlich und geduldig, und gehen auf die Zumutung des Patienten mit Engelsgeduld ein. Und siehe da: Sie werden zwar nicht immer, aber erstaunlich oft hinter der aggressiv-arroganten Fassade einen armen, hilflosen und hilfesuchenden Menschen entdecken, der sich zum dankbaren Empfänger Ihrer Hilfe durchmausert. Allerdings müssen Sie es verstehen, diese Funktion mit Taktgefühl zu übernehmen, ohne in die autoritäre Ecke abgeschoben zu werden. Das Entscheidende ist Ihre Dialogbereitschaft in einer für die konkret vorliegende Situation geeigneten Sprache.

Nochmals die Patienten. Diesmal die langweiligen Patienten. Besonders wenn Sie Spezialistin oder Spezialist geworden sind, werden Sie täglich mehrmals mit den zumindest scheinbar gleichen Krankheitsbildern konfrontiert sein. Die Routine. Die fünfte Migräne in der Sprechstunde des Kopfweh-Spezialisten. Langweilig? Oh nein! Ein Fall ist nur dann langweilig, wenn Sie selber langweilig sind. Nur dann, wenn Sie beim Fall die Krankheit und nicht den kranken Menschen sehen. Eine Krankheit spielt sich immer an einem Menschen ab, an einem Menschen in seiner individuellen, sozialen und gesellschaftlichen Einmaligkeit. Dadurch wird jeder sogenannte Fall zu etwas exquisit Einmaligem und damit Neuem und jedes Mal erneut spannend werden. Dies erfordert allerdings einige Lebendigkeit Ihrerseits, es erfordert die Bereitschaft, auf die individuellen Besonderheiten Ihres Patienten sich einzulassen, es erfordert den Mut, Abstand von der Routine zu nehmen, es erfordert Neugier Ihrerseits. Nicht Neugier im rein fachlich-medizinischen Sinn, sondern liebevolle Neugier, Bereitschaft zum persönlichen Engagement. Dann werden Sie sehen, dass auch ein Spezialgebiet trotz häufig wiederkehrender gleicher Diagnosekategorien immer wieder neu und spannend sein wird.

Apropos Spezialist. Gewiss braucht man uns Spezialisten. Wohl sehr viele unter Ihnen werden auch diesen Weg einschlagen. Vertiefte Kenntnis eines engeren Bereiches sichert grössere Kompetenz für ausgesuchte Fälle. Dies birgt allerdings auch einige Gefahren in sich. Man fühlt sich dann zwar in diesem engen Spezialbereich sehr sicher, in allem ausserhalb des eigenen Spezialgebietes aber entsprechend weniger sicher. Es besteht dann die fatale Tendenz, die eigene Aufgabe darauf zu reduzieren, dem Patienten zu sagen, ob seine Beschwerden in Ihr Spezialgebiet gehören oder nicht. Vergessen Sie bitte ob Ihrer spezialistischen Zusatzausbildung nicht Ihre allgemein-medizinische Kompetenz und im besonderen nicht Ihre ärztliche Verantwortung. Der Arzt fängt nämlich dort an, wo der Mediziner aufhört.

Der Übergang vom Studium in die Praxis lässt auch am Rande das Verhältnis zwischen Wissenschaft und den nicht wissenschaftsbezogenen Aspekten des ärztlichen Wirkens anklingen. Sie sind im Geiste sachlich-kritischer Wissenschaftlichkeit geschult worden. Das ist auch gut so. In der ärztlichen Praxis wird von Ihnen zusätzlich viel menschliche Zuwendung verlangt werden. Beide Elemente sind komplementär. Der HarvardKardiologe, Nobelpreisträger und Mitbegründer des Defibrillators, Bernard Lown, hat hierzu u.a. gesagt: «Die Fürsorge für einen Patienten ohne Wissenschaft ist zwar gutgemeinte Freundlichkeit, nicht aber gute Medizin.» Er hat dann aber hinzugefügt: «[...] andererseits beraubt eine Wissenschaft ohne Fürsorge und Anteilnahme die Medizin ihrer heilenden Fähigkeit.»

Noch ein Wort zum teuren Gesundheitswesen: Man klagt und jammert darüber, dass der Gesundheitsdienst so teuer geworden ist. Vieles ist dafür verantwortlich, und es sind daran keineswegs nur etwa die Ärzte schuld. Ganz unschuldig allerdings sind sie auch nicht. Ein Punkt, an dem Sie sparen können, sind zweifellos die Zusatzuntersuchungen, besonders die sehr kostspieligen. Versuchen Sie, wenn möglich Dinge, die klar und eindeutig sind, nicht noch zusätzlich durch eine weitere Untersuchung $\mathrm{zu}$ bestätigen. Machen Sie es sich zum Prinzip, dass Sie eine Zusatzuntersuchung nur dann anordnen sollten, wenn Sie gute Argumente dafür beibringen, dass diese Untersuchung zumindest mit dem Grade der Wahrscheinlichkeit etwas ergeben wird, was für die Patienten nützlich ist.

Und noch ein Letztes zum Ausüben der ärztlichen Tätigkeit. Gewiss haben Sie ein Anrecht darauf, durch Ihre Berufstätigkeit ein angemessenes Einkommen zu erwirtschaften. Aber vergessen wir nicht, dass der Mammon die Gefahr in sich birgt, die Moral zu korrumpieren. Wir sehen es in der Geschäftswelt und in der Welt der Politik täglich. Aber auch für den Arzt gilt dies. Für den 
Arzt reicht dies vom Durchführen nutzloser, aber gewinnbringender Untersuchungen oder Operationen über die Inrechnungstellung von nicht erbrachten Leistungen und bis hin zum Ausstellen von Gefälligkeitszeugnissen zwecks Erhaltung der Patiententreue. Lassen Sie sich nie durch den Mammon oder andere Vorteile verleiten, über wichtige Prinzipien der Moral sich hinwegzusetzen. Ohne uns mit dem heiligen Damian vergleichen zu wollen, bleibt es immerhin eine Tatsache, dass mit Recht von uns Ärzten wegen des uns entgegengebrachten Vertrauens und der in unsere Hand gegebenen Macht besonders sorgfältige Beachtung von Ethik und Moral erwartet werden. Und wenn in letzter Zeit unser Ansehen in der Gesellschaft gesunken ist, so zwar aus sehr verschiedenen und zahlreichen Gründen, aber unter anderem auch, weil man- che unter uns in einer Welt der Sachlichkeit zu locker mit den Erfordernissen der Moral und mit der Bereitschaft zu dienen umgegangen sind.

Liebe junge Kolleginnen und Kollegen, ich merke, dass meine Zeit bald abgelaufen sein wird. In diesem kirchlichen Rahmen möchte ich mich also an das 11. Gebot halten, das da lautet: Du sollst die Dir gewährte Redezeit nicht überschreiten. Lassen Sie mich also zum Abschluss Ihnen allen auch im Namen all Ihrer Lehrer und Begleiter und im Namen der hier anwesenden Angehörigen und Freunde noch einmal zum bisherigen Erfolg gratulieren und Ihnen viel Gutes für den weiteren Lebensweg wünschen. Sie haben eine schöne Zukunft vor sich, und ich kann Ihnen versichern, dass Sie den schönsten aller Berufe gewählt haben! 